\title{
ON n-VNL-modules and SVNL-modules
}

\author{
Le Cheng, Hui Wang
}

Department of Mathmatics and Computer Science, HeTao College, Bayannur, Inner Mongolia, China

Abstract: A ring R is called right n-VNL-ring if whenever $a_{1} R+a_{2} R+\cdots+a_{n} R=R$ for some elements $a_{1}, a_{2}, \cdots, a_{n} \in R$, there exists at least one element ${ }^{a_{i}}$ regular. The aim of this paper is to generalize this concept into module classes, we define $\mathrm{n}$-VNL-modules, study their properties and give some characterizations. It is proved that for any finite generated Rmodule $\mathrm{M}, \mathrm{M}$ is an SVNL-module if and only if $\mathrm{M}$ is an $\mathrm{n}$-VNL-module for every positive integer $\mathrm{n}$. The locally projective n-VNL-modules are also be characterized. We discuss the relationship between n-VNL-modules and other modules under different conditions.

Keywords: n-VNL-ring; n-VNL-module; SVNL-ring; SVNL-module

\section{Introduction}

Throughout this paper, all rings are associative with identity, all modules over a ring $\mathrm{R}$ are right $\mathrm{R}$-modules.

An element $a$ of ring $R$ is called regular if $a=a b a$ for some $b \in R$. A ring $R$ is regular if all of its elements are regular. A ring $\mathrm{R}$ is local if it has only one maximal ideal. The generalization of regular rings and local rings is one of the most popular topics in the research of ring theory, and grow out many new subjects, in which the n-VNL-rings and SVNL-rings play important roles. Contessa called a ring $\mathrm{R}$ is VNL-ring if a or $1-\mathrm{a}$ is regular for every $a \in R$. Osba called a commutative ring $\mathrm{R}$ is SVNL-ring if whenever $\langle S\rangle=R$ for some nonempty subset $\mathrm{S}$ of $\mathrm{R}$, there exists at least one element $s \in S$ regular, where $\langle S\rangle$ is an ideal generated by $\mathrm{S}$. Chen and Ying called a ring $\mathrm{R}$ is right $\mathrm{n}$-VNL if whenever $a_{1} R+a_{2} R+\cdots+a_{n} R=R$ for some elements $a_{1}, a_{2}, \cdots, a_{n} \in R$, there exists at least one element is regular. Left $\mathrm{n}$-VNLrings are defined analogously, and a ring $\mathrm{R}$ is a $\mathrm{n}$-VNL-ring if it is a left and right $\mathrm{n}$-VNL-ring.

Introducing the concept of rings into modules is a common method to generalize ring classes. We have introduced the concept of SVNL-ring into module classes, a module M over a ring R is called SVNL-module if whenever $\langle S\rangle=M$ for some nonempty subset $\mathrm{S}$ of $\mathrm{M}$, then there exists at least one element $s \in S$ such that sR is a direct summand of M. The aim of this paper is to generalize the concepts of n-VNL-ring to module classes, study their properties and give some characterizations.

\section{2. n-VNL-Modules}

Definition: A module M over a ring R is called n-VNL-module if whenever $s_{1} R+s_{2} R+\cdots+s_{n} R=M$ for some

Copyright $(2020$ by author(s) and Frontier Scientific Research Publishing Inc.

This work is licensed under the Creative Commons Attribution International License (CC BY 4.0)

http://creativecommons.org/licenses/by/4.0/ 
elements $s_{1}, s_{2}, \cdots, s_{n} \in M$, there exists $s_{i}$ such that $s_{i} R$ is a direct summand of M.

2.1 Proposition: Let $\mathrm{R}$ be a ring, and then the following conclusions are established:

(1) $\mathrm{R}$ is a right $\mathrm{n}-\mathrm{VNL}$-ring if and only if RR is a $\mathrm{n}-\mathrm{VNL}-$ module;

(2) Every module is 1 -VNL-module. If $n \geq m \geq 1$, a n-VNL-module must be a m-VNL-module;

(3) A SVNL-module must be a n-VNL-module for every positive integer $n$;

(4) A non-finite generated module must be a n-VNL-module for every positive integer n;

(5) A NJ-module must be a n-VNL-module for every positive integer $n$.

Proof: According to the definition of n-VNL-module, (1) (2) (3) (4) are obvious. We just need to prove (5).

A module MR is called NJ module if $x R$ is a direct summand of $\mathrm{M}$ for every element $x \in M / J(M)$. If MR is a NJ module and $s_{1}, s_{2}, \cdots, s_{n} \in M$ such that $s_{1} R+s_{2} R+\cdots+s_{n} R=M$, then there must exit an element $s_{i} \notin J(M)$, so $s_{i} R$ is a direct summand of $\mathrm{M}$. Therefore, MR is a $\mathrm{n}-\mathrm{VNL}-$ module.

Example: (1) Let $\mathrm{p}$ be a prime number, $M=\left\{a / p^{n} \mid a \in \mathrm{Z}, n \in \mathrm{N}\right\}$ is a subset of rational number set. We use the mark $\mathrm{Z}_{p^{\infty}}$ to represent the quotient module $M / \mathrm{Z}$, then $\mathrm{Z}_{p^{\infty}}$ is a non-finite generated module as a $\mathrm{Z}$-module, so $\mathrm{Z}_{p^{\infty}}$ is a nVNL-module for every positive integer $n$.

If $\mathrm{R}$ is a commutative ring, Chen and Tong have proved $\mathrm{R}$ is a VNL-ring if and only if it is an SVNL-ring. Chen and Ying proved every right 2-VNL-ring $\mathrm{R}$ is VNL-ring. Osba proved $Z_{n}$ is SVNL-ring if and only if $(p q)^{2} \mid R_{\text {for any prime }}$ number $\mathrm{p}$ and $\mathrm{q}$. In fact, for any subset $\mathrm{A}$ of $Z_{n}$, the submodule of $\left(Z_{n}\right)_{Z}$ which generated by $A$ is the same as the submodule of $\left(Z_{n}\right)_{Z_{n}}$ generated by A. So We have the following conclusion:

(2) $Z_{n}$ is 2-VNL as a Z-module if and only if $(p q)^{2} \mid \mathbb{R}$ for any prime number $\mathrm{p}$ and $\mathrm{q}$.

2.2 Proposition: Let $\mathrm{R}$ be a ring, $\mathrm{M}$ is a finite generated $\mathrm{R}$-module, then the following are equivalent:

(1) $\mathrm{M}$ is a SVNL-module;

(2) $\mathrm{M}$ is a n-VNL-module for every positive integer $n$.

Proof: The prove (1) $\Rightarrow(2)$ is obvious, we just need to prove (2) $\Rightarrow$ (1). Let M be a n-VNL-module for every positive integer n, and $S \subseteq M$ which satisfy $\langle S\rangle=M$. Since $\mathrm{M}$ is a finite generated module, there exists some elements $s_{1}, s_{2}, \cdots, s_{n} \in M$, such that:

$$
s_{1} R+s_{2} R+\cdots+s_{n} R=M
$$

Because $\mathrm{M}$ is a n-VNL-module, there exists an element $s_{i} \in S$ such that $S_{i} R$ is a direct summand of $\mathrm{M}$, so $\mathrm{M}$ is a SVNL-module.

Its need to be notice that the condition "finite generated" is necessary. For example, $\mathrm{Z}_{p^{\infty}}$ is n-VNL-module as a Zmodule, but every submodule of $\mathrm{Z}_{p^{\infty}}$ is cyclic module and generated by $1 / p^{n}\left(n \geq 1, p_{\text {is a prime number), so any submodule }}\right.$ of $\mathrm{Z}_{p^{\infty}}$ can not be direct summand of $\mathrm{Z}_{p^{\infty}}$, that means $\mathrm{Z}_{p^{\infty}}$ is not a SVNL-module.

Next, we will gave a sufficient condition for $n-V N L-m o d u l e$. 
2.3 Proposition: Let $\mathrm{R}$ be a ring, $\mathrm{n}$ is a positive integer, if $M_{R}$ is a module which satisfied for any full homomorphism $f: R^{n} \rightarrow M$, there must exist a number $\mathrm{j}(1 \leq j \leq n)$ such that homomorphism $f_{j}=f i_{j}: R \rightarrow M$

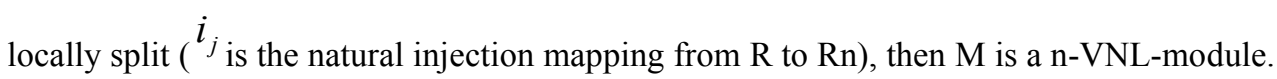

Proof: Suppose $s_{1}, s_{2}, \cdots, s_{n} \in M$, we can define a cluster R-homomorphism:

$$
f_{k}: R \rightarrow M, f_{k}(1)=s_{k}, 1 \leq k \leq n .
$$

If $s_{1} R+s_{2} R+\cdots+s_{n} R=M$, then the homomorphism $f=\oplus_{k=1}^{n} f_{i}: R^{(n)} \rightarrow M$ is full, so there exists a number $\mathrm{j}$ $(1 \leq j \leq n)_{\text {such that homomorphism }} f_{j}=f i_{j}: R \rightarrow M$ locally split. For $s_{j}=f_{j}(1) \in f_{j} R$, there exists a homomorphism $g: M \rightarrow R$ such that:

$$
s_{j}=f_{j} g\left(s_{j}\right)=f_{j}(1) g\left(s_{j}\right)=s_{j} g\left(s_{j}\right)
$$

Suppose $^{h=f_{j} g}$, then

$$
h\left(s_{j}\right)=f_{j} g\left(s_{j}\right)=s_{j} g\left(s_{j}\right)=s_{j} .
$$

This means $h$ is locally split, and $s_{j} R$ is a direct summand of M. Hence M is a n-VNL-module.

Now we can give some equivalent characterizations of locally projective n-VNL-module.

2.4 Theorem: Let $M$ be a locally projective module, for every positive integer $n$, the following statements are equivalent:

(1) $\mathrm{M}$ is a n-VNL-module;

(2) For any full homomorphism $f: R^{n} \rightarrow M$, there exists a number $\mathrm{j}(1 \leq j \leq n)$ such that homomorphism $f_{j}=f i_{j}: R \rightarrow M$ locally split;

(3) If some elements $S_{1}, S_{2}, \cdots, s_{n}$ in M satisfy $s_{1} R+s_{2} R+\cdots+s_{n} R=M$, then there exist at least one element $S_{i}$ such that $S_{i} R$ is a projective direct summand of $\mathrm{M}$.

Proof: Since a finite generated locally projective module is a projective module, so (1) $\Rightarrow(3)$ and (3) $\Rightarrow(1)$ is obvious.

(1) $\Rightarrow$ (2) Let $f: R^{n} \rightarrow M$ be a full homomorphism, we define a set $\mathrm{S}$ as follow:

$$
S=\left\{y_{k}=f\left(x_{k}\right) \mid x_{k} \in R^{n} ; \pi\left(x_{k}\right)=0(j \neq k), 1 \leq j, k \leq n\right\}
$$

where $\pi_{k}$ is the natural full homomorphism from $R^{n}$ to $R$. It is easy to know $S \subseteq M$ and $\langle S\rangle=M$, so M is finite generated. By proposition 2.2, $\mathrm{M}$ is SVNL-module, so there exists a number $\mathrm{j}(1 \leq j \leq n)$ such that $y_{i} R$ is a direct summand of $\mathrm{M}$, next we prove the homomorphism $f_{j}=f i_{j}: R \rightarrow M$ locally split.

$\mathrm{M}$ is a projective module, $y_{i} R$ is a direct summand of $\mathrm{M}$, so $y_{i} R$ is also a projective module. Hence the mapping $h: R \rightarrow y_{j} R$ is locally split, there exists homomorphism $q: y_{j} R \rightarrow R$ such that $y_{j}=h q\left(y_{j}\right)=h(1) q\left(y_{j}\right)=y_{j} q\left(y_{j}\right)$. 
Let $g=q \pi_{j}$, then for any element $y_{j} r \in f_{j} R=y_{j} R$, we have:

$$
f_{j} g\left(y_{j} r\right)=f_{j}(1) q\left(y_{j} r\right)=y_{j} r
$$

It means $f_{j}=f i_{j}: R \rightarrow M$ is locally split.

(2) $\Rightarrow$ (1) By proposition 2.3, it's obvious.

\section{Relationship between Some Module Classes}

In the last part of this article, we summarize the relationship among the ring classes which related to n-VNL-rings, and the relationship among the module classes which related to n-VNL-modules.

To generalize regular and local rings, Contessa, Osba, Chen and Nicholson defined and discussed VNL-ring, SVNLring, $\mathrm{n}$-VNL-ring and $\mathrm{NJ}$ ring respectively. According to their conclusion, the relationship among these kinds of rings can be describe as follow:

$$
\text { Regular ring (or local ring) } \Rightarrow \text { NJ ring } \Rightarrow \text { SVNL ring } \Rightarrow_{\mathrm{n}-\mathrm{VNL}-\text { ring }(\mathrm{n} \geq 1)}
$$

The arrow above means roll out, and reverse the arrows are all invalid.

Roger Ware has introduced the concept of regular ring and local ring into module classes, he defined regular module and local module. Zelmanowitz and Azumaya also used another different method to define regular modules. We have introduced the concept of NJ ring and SVNL-ring into module classes, defined NJ module, SNJ module and SVNL module. According to the conclusion, the relationship among these modules under different conditions can be describe as follow:

Corollary 1: Under normal condition, the relationship among the module classes which related to n-VNL-modules can be expressed as follow:

$$
\begin{aligned}
& \text { Rugular module (or local module) } \Rightarrow \text { SVNL module } \Rightarrow \mathrm{n} \text { - VNL - module }(\mathrm{n} \geq 1) \\
& \qquad \\
& \text { SNJ module } \Rightarrow \mathrm{NJ} \text { module } \Rightarrow \mathrm{n} \text { - VNL - module }(\mathrm{n} \geq 1)
\end{aligned}
$$

Corollary 2: Under the finite generated condition, the relationship among the module classes which related to n-VNLmodules can be expressed as follow:

$$
\begin{array}{r}
\text { Rugular module (or local module) } \Rightarrow \mathrm{NJ} \text { module } \Rightarrow \text { SVNL module } \\
\Downarrow \\
n-\text { VNL - module }(n \geq 1)
\end{array}
$$

Corollary3: Under the locally projective condition, the relationship among the module classes which related to $\mathrm{n}$ VNL-modules can be expressed as follow:

$$
\begin{array}{r}
\text { Rugular module (or local module) } \Rightarrow \mathrm{SNJ} \text { module } \Leftrightarrow \mathrm{NJ} \text { module } \Rightarrow \mathrm{SVNL} \text { module } \\
\Downarrow \\
n \text {-VNL-module }(n \geq 1)
\end{array}
$$

\section{Conflicts of Interest}

The author declares no conflicts of interest regarding the publication of this paper.

\section{Acknowledgments}

This research was supported by the Natural Science Foundations of HeTao College (HYZQ201945): Research on Generalization and Application of VNL Rings.

\section{References}

[1] Contessa M. (1984). On Certain Classes of PM-rings. Communications in Algebra, (12): 1447-1469. 
[2] Osba E.A., Henriksen M. and Alkam O. (2004). Combining Local and Von Neumann Regular Rings. Communications in Algebra, (32): 2639-2653.

[3] Chen J.L. and Ying Z.L. (2008). On VNL-rings and n-VNL-rings. International Electronic Journal of Algebra, (4): $1-8$.

[4] Cheng L., Yang S.S. and Zhang X.J. (2015). Generalization of Strongly Von Neumann Local Ring. Journal of HuaiHai Institute of Technology, 100(2): 1-3.

[5] Cheng L. and Chen J.L. (2015). NJ Modules and SNJ Modules. Journal of NanJing University (Mathematical Biquarterly), 032(001): 89-95.

[6] Chen W.X. and Tong W.T. (2006). On Noncommutative VNL-rings and GVNL Rings. Glasgow Math, (48): 1117.

[7] Nicholson W.K. (1973). Rings Whose Elements are Quasi-regular or Regular. Aequationes Mathematicae, (9): 6470 .

[8] Roger W. (1972). Endomorphism Rings of Projective Modules. Transactions of the American Mathematical Society, (155): 233-256.

[9] Zelmanowitz J. (1972). Regular Modules. Transactions of the American Mathematical Society, (163): 341-355.

[10] Goro A. (1990). Some Characterizations of Regular Modules. Publications Matemàtiques, (34): $241-248$.

[11] Jayaraman M. and Vanaja N. (2007). Generalization of Regular Modules. Communications in Algebra, (35): $3331-3345$. 